\title{
Determinantes da autopercepção de saúde entre idosos do Município de São Paulo, Brasil ${ }^{1}$
}

\author{
Luciana Correia Alves $^{2}$ e Roberto Nascimento Rodrigues ${ }^{2}$
}

Como citar

Alves LS, Rodrigues RN. Determinantes da autopercepção de saúde entre idosos do Município de São Paulo, Brasil. Rev Panam Salud Publica. 2005;17(5/6):333-41.

RESUMO Objetivo. Investigar a influência de determinantes demográficos e socioeconômicos, das doenças crônicas e da capacidade funcional sobre a autopercepção de saúde entre os idosos do Município de São Paulo e verificar a existência de diferenças entre os sexos quanto à autopercepção de saúde. Métodos. O estudo foi desenvolvido com base em dados do Projeto Saúde, Bem-Estar e Envelhecimento na América Latina e Caribe (SABE). Foram analisados os dados de 2135 idosos (sendo 58,6\% mulheres; idade média e mediana = 69,4 e 68,0 anos). A variável dependente foi a autopercepção de saúde (ruim ou boa). As variáveis independentes foram: as demográficas (idade, sexo, estado conjugal e arranjo familiar), as socioeconômicas (educação e renda), o número de doenças crônicas (hipertensão, artrite ou reumatismo, doença cardiovascular, diabetes, asma, bronquite ou enfisema, embolia ou acidente vascular cerebral e câncer) e a capacidade funcional. Para estimar a associação entre a autopercepção de saúde e as variáveis independentes e estudar as diferenças entre os sexos, foi realizada uma análise de regressão logística binária múltipla.

Resultados. A presença de doenças crônicas associada ao sexo do idoso foi o determinante mais fortemente relacionado à autopercepção de saúde no Município de São Paulo. Para os homens, a presença de quatro ou mais doenças crônicas implicou um risco 10,53 vezes maior de uma autopercepção ruim de saúde. Para as mulheres, esse risco foi 8,31 vezes maior. A capacidade funcional, o nível de escolaridade e a renda também foram altamente associados com a percepção de saúde, e a idade teve uma significativa influência. Na ausência de doenças crônicas, ou na presença de duas ou mais doenças crônicas, as mulheres idosas tiveram maior probabilidade de relatar uma boa autopercepção de saúde em comparação com os homens.

Conclusões. Os resultados indicam a necessidade de ações integradas que abordem simultaneamente os principais fatores determinantes da autopercepção de saúde como forma de promover o bem-estar e a qualidade de vida dos idosos.

Palavras-chave Doenças crônicas, fatores sexuais, saúde do idoso.

Desde as últimas décadas do século passado, o Brasil se depara com um declínio rápido e acentuado da fecun-

1 Trabalho baseado em dissertação de mestrado apresentada ao Curso de Pós-Graduação em Demografia do Centro de Desenvolvimento e Planejamento Regional (CEDEPLAR) da Universidade Federal de Minas Gerais (UFMG).

2 UFMG, CEDEPLAR, Departamento de Demografia. Enviar correspondência para Luciana Correia didade, fenômeno sem precedentes em sua história. Como aconteceu na maioria dos países em desenvolvimento, esse declínio, combinado com a

Alves no seguinte endereço: Rua José Oscar Barreira 104, Itapoã, CEP 31710-580, Belo Horizonte, MG, Brasil. Fone: +55-31-3491-9470; fax: +55-313201-3657; e-mail: lcalves@cedeplar.ufmg.br e lucianacalves@ig.com.br queda da mortalidade, acarretou um processo de envelhecimento populacional. Em 2000, a proporção de pessoas com 60 anos ou mais no Brasil correspondia a $8,6 \%$ da população total, em comparação com $7,3 \%$ no ano de 1991 (1). As projeções indicam que, em 2020, a população de idosos no Brasil será de mais de 26,2 milhões de indiví- 
duos, representando quase $12,4 \%$ da população total. ${ }^{3}$

O crescimento da população idosa brasileira pode representar um grave problema para a sociedade se os anos de vida adicionais não forem vividos em condições de saúde adequadas (2). A saúde é fundamental para que os idosos se mantenham independentes e autônomos e continuem contribuindo para a sociedade (3). Na ausência de políticas públicas adequadas, a tendência é que se tenha, no Brasil, um número crescente de indivíduos idosos que, mesmo vivendo mais, apresentem uma saúde precária e sejam funcionalmente incapacitadosou seja, sejam incapazes de executar tarefas cotidianas (4).

A caracterização das condições de saúde dos idosos requer informações detalhadas sobre diferentes aspectos da vida desses indivíduos (5). Nos países desenvolvidos, é crescente o número de investigações que abordam as associações entre a saúde dos idosos e os determinantes demográficos e socioeconômicos, as doenças crônicas e a capacidade funcional. Uma forma de conduzir esse tipo de estudo é o levantamento de informações sobre a percepção dos idosos em relação ao seu próprio estado de saúde. Embora a mensuração do estado de saúde seja bastante difícil, já que engloba diversos aspectos da vida do indivíduo, a autopercepção de saúde tem se mostrado um método confiável, e, recentemente, mais utilizado do que a observação direta para a análise desse aspecto (6-8).

A autopercepção de saúde contempla aspectos da saúde física, cognitiva e emocional (9). Além disso, é um poderoso indicador de mortalidade (10, 11), ou seja: as pessoas que relatam a sua saúde como sendo pobre apresentam maior risco de mortalidade por todas as causas de morte em comparação com aquelas que relatam ter uma saúde excelente (12). A autoper-

\footnotetext{
3 Universidade Federal de Minas Gerais, Centro de Desenvolvimento e Planejamento Regional. Projeção populacional das unidades da Federação, Brasil, por sexo e grupos qüinqüenais de idade: 1990-2020 [documento mimeografado]. Belo Horizonte: UFMG/CEDEPLAR; 1999.
}

cepção associa-se fortemente com o estado real ou objetivo de saúde das pessoas e pode ser encarada como uma representação das avaliações objetivas de saúde (13).

Um envelhecimento saudável depende da interação multidimensional de vários fatores. No entanto, são poucos os trabalhos que exploram um modelo que combine a idade, o sexo, o arranjo familiar, o estado conjugal, a educação, a renda, as doenças crônicas e a capacidade funcional, especialmente nos países em desenvolvimento, como é o caso específico do Brasil (14).

Assim, o objetivo deste estudo foi investigar a influência de determinantes demográficos e socioeconômicos, de certas doenças crônicas e da capacidade funcional sobre a autopercepção de saúde entre os idosos do Município de São Paulo, e verificar a existência de diferenças entre os sexos quanto à autopercepção de saúde.

\section{MATERIAIS E MÉTODOS}

O estudo foi desenvolvido com base em dados oriundos do Projeto Saúde, Bem-Estar e Envelhecimento na América Latina e Caribe (SABE) (15). O SABE é um estudo epidemiológico transversal, de base populacional, domiciliar, realizado em sete países da América Latina e Caribe, sob a coordenação da Organização Pan-Americana da Saúde (OPAS). No Brasil, o SABE circunscreveu-se aos limites territoriais do Município de São Paulo. Foram entrevistados 2143 idosos (indivíduos com 60 anos ou mais) nãoinstitucionalizados, de ambos os sexos, no período entre janeiro de 2000 e março de 2001.

A vantagem do Projeto SABE em relação às outras pesquisas que abordam as condições de saúde no Brasil é que, além de ser um estudo específico de saúde, direcionado exclusivamente aos indivíduos de 60 anos ou mais, ele contém informações mais aprofundadas do que as de outros estudos sobre vários domínios da saúde e sobre fatores diretamente relacionados à saúde. A capacidade funcional, por exemplo, é avaliada detalhadamente, por meio de três componentes: atividades de vida diária (seis itens), atividades instrumentais de vida diária (nove itens) e mobilidade (12 itens). Em contrapartida, a Pesquisa Nacional por Amostra de Domicílios (PNAD-98) (16), embora apresente a vantagem de ser representativa para o conjunto do Brasil, para as unidades da federação e para as regiões metropolitanas, não investiga as atividades instrumentais de vida diária e analisa somente três itens das atividades de vida diária (alimentar-se, tomar banho e ir ao banheiro).

O SABE tomou como referência o desenho amostral da PNAD-98. ${ }^{4} \mathrm{O}$ processo de amostragem foi realizado em dois estágios: no primeiro, selecionaram-se os setores censitários e, no segundo, foram selecionados os domicílios dentro de cada setor. Em cada domicílio foram entrevistadas todas as pessoas residentes com 60 anos ou mais, independentemente do seu estado conjugal ou grau de parentesco. O presente estudo selecionou os dados levantados pelo $\mathrm{SABE}$ relativos a todos os indivíduos com 60 anos ou mais que responderam à pergunta referente à auto-avaliação do estado de saúde. Foram excluídos dados relativos a oito idosos que deixaram de fornecer informações sobre as variáveis enfocadas neste estudo. Assim, a amostra considerada foi constituída de 2135 idosos.

Entre os indivíduos incluídos no estudo, 87,2\% responderam eles próprios a toda a entrevista. As demais entrevistas foram respondidas apenas parcialmente pelo entrevistado ou foram inteiramente respondidas por um outro informante. Dos participantes do estudo, $58,6 \%$ eram do sexo feminino e $41,4 \%$ do sexo masculino. A idade variou de 60 a 100 anos, sendo que a idade média e a idade mediana foram de 69,4 e 68,0 anos, respectivamente.

A variável dependente foi a autopercepção de saúde. Neste estudo, essa medida foi dicotomizada em autopercepção boa ou ruim de saúde. A

\footnotetext{
4 Palloni A, Peláez M. Survey on health and wellbeing of elders [documento mimeografiado] Washington, D.C.: Pan American Health Organization; 2002.
} 
combinação das categorias excelente, muito boa e boa indicou uma boa autopercepção de saúde, e a combinação das categorias regular e ruim indicou uma autopercepção ruim de saúde.

Sabe-se que a percepção de saúde envolve aspectos subjetivos e, portanto, é de se esperar que haja diferenças entre a percepção baseada nas respostas do próprio idoso ou nas respostas relatadas parcial ou totalmente por outro informante. Por isso, foi criada a variável "informante", que identifica se as respostas foram ou não fornecidas totalmente pelo idoso de referência. Como o questionário do SABE não identificava exatamente quem respondeu à entrevista, a variável informante foi construída com base na avaliação do estado cognitivo do idoso. No SABE, foi utilizado o questionário Annotated Mini Mental State Examination (AMMSE) (17) modificado por Icaza e Albala (18) para investigar o estado mental do indivíduo. Um ponto de corte de 12 pontos foi aplicado para selecionar os idosos com um visível comprometimento cognitivo. Assim, os indivíduos que alcançaram um escore de 12 ou menos foram classificados como tendo déficit cognitivo. Os idosos que obtiveram uma pontuação de 13 ou mais no teste foram considerados como capazes de responder a entrevista independentemente. Quando o indivíduo pontuou 12 ou menos no AMMSE, o Portable Functional Assessment Questionnaire (escala Pfeffer) (19) foi administrado a uma outra pessoa que residia no domicílio e que poderia ajudar a responder a entrevista. Assim, foi possível indicar se houve a participação de um segundo indivíduo na entrevista, e a variável informante foi codificada como 0 (outro informante) ou 1 (entrevistado).

As variáveis independentes foram classificadas em quatro grupos: o demográfico (idade, sexo, estado conjugal e arranjo familiar), o socioeconômico (educação e renda), o do número de doenças crônicas (hipertensão, artrite ou reumatismo, doença cardiovascular, diabetes, asma, bronquite ou enfisema, embolia ou acidente vascular cerebral e câncer), e o da capacidade funcional (atividades de vida diária: atravessar um cômodo da casa, comer, deitar-se e levantar da cama, usar o vaso sanitário, vestir-se e despir-se e tomar banho; e atividades instrumentais de vida diária: preparar uma refeição quente e realizar tarefas domésticas leves e pesadas). Os idosos foram classificados como sendo dependentes quando relataram necessidade de ajuda para realizar pelo menos uma das atividades relacionadas a cada dimensão. Uma escala de incapacidade hierárquica foi construída distinguindo três categorias: 1) indivíduo independente; 2) indivíduo dependente nas atividades instrumentais de vida diária; e 3) indivíduo dependente nas atividades de vida diária e nas atividades instrumentais de vida diária. Os idosos que relataram dependência nas atividades de vida diária, mas não nas atividades instrumentais, foram classificados na categoria 3 .

No SABE, a população de 75 anos ou mais foi sobreamostrada, e esse problema foi corrigido por meio dos pesos amostrais, o que requer sua utilização para a estimativa de indicadores. Assim, para garantir a representatividade da população total, foram incorporados aos dados os pesos para expansão da amostra, o que possibilitou a obtenção de estimativas nãoviciadas para os parâmetros populacionais. Contudo, as estimativas de medidas de dispersão não são obtidas de forma adequada com a simples utilização dos pesos. Esse é o caso do erro padrão associado com os coeficientes das variáveis usadas no modelo de regressão. As estimativas dos coeficientes são influenciadas apenas pelos pesos, ao passo que as estimativas das medidas de dispersão são influenciadas também pela estratificação e conglomeração. Sendo assim, seria preciso incorporar, no processo de estimação dessas medidas, os aspectos que definem o plano amostral complexo. Embora certos programas de computador, como o SUDAAN, permitam a incorporação de alguns planos amostrais, nenhum programa consegue incorporar o plano amostral do $\mathrm{SABE}$, que é uma subamostra da PNAD.

Para estimar a associação entre a autopercepção de saúde e cada variável demográfica e socioeconômica, o número de doenças crônicas e a capacidade funcional, e para estudar os diferenciais por sexo, foi realizada uma análise de regressão logística binária múltipla. As variáveis foram incorporadas uma a uma de forma seqüencial no modelo.

Os resultados dos modelos foram apresentados como razão de chances (odds ratio, OR), tendo como referência intervalos de confiança de $95 \%$ (IC95\%). As variáveis sexo e informante, que controlam o efeito produzido pelas respostas dadas pelo próprio entrevistado, foram consideradas como variáveis de confundimento neste estudo e foram incluídas em todos os modelos logísticos.

\section{RESULTADOS}

A tabela 1 apresenta os parâmetros da regressão logística binária para a autopercepção ruim de saúde dos indivíduos idosos. Embora os ajustes dos modelos de regressão devam ser feitos na escala logito, tecnicamente mais apropriada, a interpretação substantiva, neste estudo, está apresentada em OR, tendo em vista que essa forma é de mais fácil comparação e compreensão (tabela 2).

No modelo 1 da tabela 2, nota-se que, entre os indivíduos de 60 anos ou mais, a chance de auto-avaliar a saúde como ruim foi $16 \%$ maior para as mulheres do que para os homens. Além disso, a chance de auto-avaliar a saúde como ruim foi $47 \%$ menor quando as informações foram dadas pelo próprio idoso em comparação com idosos cuja avaliação foi respondida por outro informante.

O modelo 2 (tabela 2) evidencia que a idade é um importante determinante da autopercepção de saúde. As chances de os idosos relatarem uma saúde negativa foram maiores do que na categoria de referência em todos os grupos etários, mas declinaram com o avançar da idade. A entrada da idade no modelo não modificou o efeito das variáveis sexo e informante.

O modelo 3 (tabela 2) mostra que o arranjo familiar tem uma grande força 
TABELA 1. Parâmetros estimados pela regressão logística binária para autopercepção ruim de saúde em idosos do Município de São Paulo (SP), Brasil, 2000 e 2001

\begin{tabular}{|c|c|c|c|c|c|c|c|c|}
\hline & Modelo 1 & Modelo 2 & Modelo 3 & Modelo 4 & Modelo 5 & Modelo 6 & Modelo 7 & Modelo 8 \\
\hline \multicolumn{9}{|l|}{ Informante } \\
\hline Entrevistado & $-0,638^{a}$ & $-0,670^{a}$ & $-0,659^{a}$ & $-0,667^{a}$ & $-0,540^{a}$ & $-0,503^{a}$ & $-0,519^{a}$ & $-0,197^{a}$ \\
\hline \multicolumn{9}{|l|}{ Sexo } \\
\hline Homens & 0,000 & 0,000 & 0,000 & 0,000 & 0,000 & 0,000 & 0,000 & 0,000 \\
\hline Mulheres & $0,146^{\mathrm{a}}$ & $0,148^{\mathrm{a}}$ & $0,179^{a}$ & $0,195^{\mathrm{a}}$ & $0,165^{a}$ & $0,152^{\mathrm{a}}$ & $-0,008$ & $-0,094^{\mathrm{a}}$ \\
\hline 65 a 69 & & $0,331^{\mathrm{a}}$ & $0,342^{\mathrm{a}}$ & $0,347^{a}$ & $0,373^{a}$ & $0,344^{a}$ & $0,235^{a}$ & $0,237^{a}$ \\
\hline 70 a 74 & & $0,167^{a}$ & $0,190^{\mathrm{a}}$ & $0,202^{\mathrm{a}}$ & $0,171^{\mathrm{a}}$ & $0,126^{a}$ & $-0,029^{a}$ & $-0,074^{\mathrm{a}}$ \\
\hline 75 a 79 & & $0,290^{\mathrm{a}}$ & $0,334^{a}$ & $0,350^{\mathrm{a}}$ & $0,324^{a}$ & $0,254^{\mathrm{a}}$ & $0,099^{a}$ & $0,055^{\mathrm{a}}$ \\
\hline 80 ou mais & & $-0,010$ & $0,036^{a}$ & $0,063^{a}$ & 0,014 & $-0,036^{a}$ & $-0,199^{a}$ & $-0,392^{\mathrm{a}}$ \\
\hline \multicolumn{9}{|l|}{ Arranjo familiar } \\
\hline Mora acompanhado & & & 0,000 & 0,000 & 0,000 & 0,000 & 0,000 & 0,000 \\
\hline Viúvo & & & & 0,020 & $-0,067^{a}$ & $-0,085^{a}$ & $-0,141^{a}$ & $-0,132^{\mathrm{a}}$ \\
\hline \multicolumn{9}{|l|}{ Escolaridade } \\
\hline Sem escolaridade & & & & & 0,000 & 0,000 & 0,000 & 0,000 \\
\hline 1 a $4^{a}$ série (ensino fundamental) & & & & & $-0,450^{\mathrm{a}}$ & $-0,390^{\mathrm{a}}$ & $-0,349^{a}$ & $-0,329^{\mathrm{a}}$ \\
\hline $5^{\mathrm{a}}$ série ou mais (ensino fundamental) & & & & & $-1,142^{\mathrm{a}}$ & $-1,023^{a}$ & $-0,979^{a}$ & $-0,968^{\mathrm{a}}$ \\
\hline \multicolumn{9}{|l|}{ Renda (salários mínimos) ${ }^{b}$} \\
\hline$<1$ & & & & & & 0,000 & 0,000 & 0,000 \\
\hline 1 a 3 & & & & & & $0,352^{\mathrm{a}}$ & $0,295^{a}$ & $0,284^{\mathrm{a}}$ \\
\hline 3 a 5 & & & & & & $0,126^{a}$ & $-0,021^{\mathrm{c}}$ & $-0,016$ \\
\hline 5 ou mais & & & & & & $-0,292^{\mathrm{a}}$ & $-0,411^{a}$ & $-0,392^{\mathrm{a}}$ \\
\hline \multicolumn{9}{|l|}{ Número de doenças crônicas ${ }^{d}$} \\
\hline Nenhuma & & & & & & & 0,000 & 0,000 \\
\hline 1 & & & & & & & $0,621^{a}$ & $0,610^{\mathrm{a}}$ \\
\hline
\end{tabular}

Fonte dos dados básicos: Projeto Saúde, Bem-Estar e Envelhecimento na América Latina e Caribe (SABE) (15).

a $P<0,01$.

b Salário mínimo em abril de 2000: R\$ 151 (US\$ 89,93).

${ }^{c} P<0,05$.

${ }^{d}$ Hipertensão, artrite ou reumatismo, doença cardiovascular, diabetes, asma, bronquite ou enfisema, embolia ou acidente vascular cerebral e câncer.

e AIVD = atividades instrumentais de vida diária (preparar uma refeição quente e realizar tarefas domésticas leves e pesadas); AVD = atividades de vida diária (atravessar um cômodo da casa, comer, deitar-se e levantar da cama, usar o vaso sanitário, vestir-se e despir-se e tomar banho).

explicativa na autopercepção de saúde. O idoso que mora sozinho avalia a sua saúde mais positivamente em relação àqueles que moram acompanhados, mesmo depois do controle pelas variáveis informante, sexo e idade. $\mathrm{O}$ arranjo familiar contribuiu para o aumento nos diferenciais entre os sexos quanto à percepção ruim de saúde. Quando o estado conjugal foi inserido, no modelo 4 , as desigualdades entre os sexos quanto à autopercepção de saúde aumentaram ainda mais. A chance de as mulheres idosas declararem uma saúde ruim foi $22 \%$ maior em comparação aos homens idosos. Os idosos separados ou divorciados e aqueles casados ou em união consensual apresentaram uma chance 12 e 9\% maior, respectivamente, de perceber a própria saúde como ruim em relação aos solteiros (categoria de referência).
O nível de escolaridade, introduzido no modelo 5 (tabela 2), apresentou-se como um importante fator de proteção de uma percepção ruim de saúde entre os idosos após controle pela variável informante e pelas variáveis demográficas. A introdução da escolaridade no modelo reduziu as diferenças entre os sexos quanto à autopercepção de uma saúde ruim. Neste modelo, as chances de as mulheres avaliarem a sua saúde 
TABELA 2. Razão de chances (OR) dos modelos logísticos binários para autopercepção ruim de saúde em idosos do Município de São Paulo (SP), Brasil, 2000 e 2001

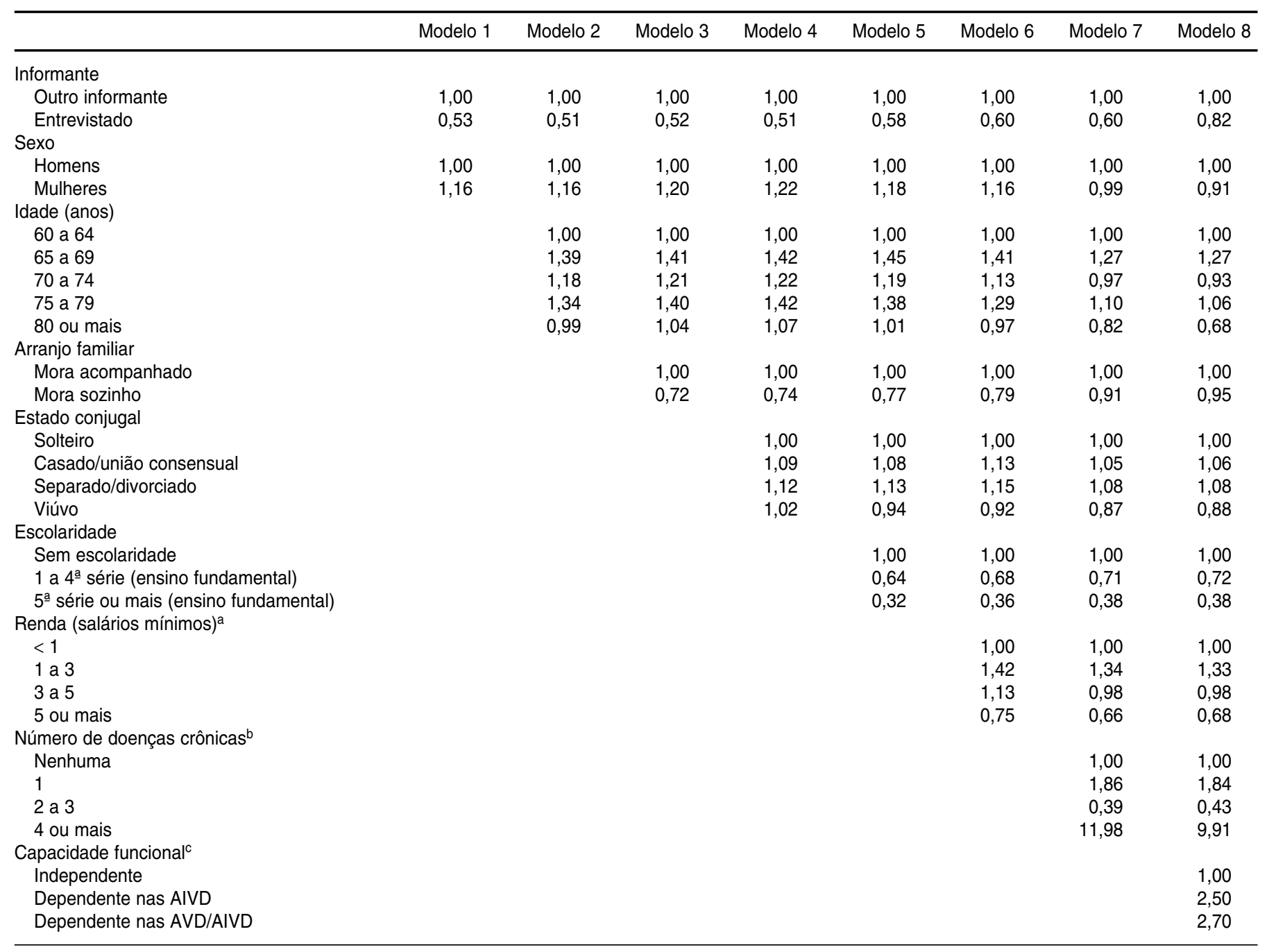

Fonte dos dados básicos: Projeto Saúde, Bem-Estar e Envelhecimento na América Latina e Caribe (SABE) (15).

a Salário mínimo em abril de 2000: R\$151 (US\$ 89,93).

b Hipertensão, artrite ou reumatismo, doença cardiovascular, diabetes, asma, bronquite ou enfisema, embolia ou acidente vascular cerebral e câncer.

${ }^{\mathrm{C}} \mathrm{AIVD}$ = atividades instrumentais de vida diária (preparar uma refeição quente e realizar tarefas domésticas leves e pesadas); AVD = atividades de vida diária (atravessar um cômodo da casa, comer, deitar-se e levantar da cama, usar o vaso sanitário, vestir-se e despir-se e tomar banho).

de maneira negativa foram $18 \%$ maiores em comparação com os homens.

A renda também foi significativamente associada com a autopercepção de saúde entre os idosos (modelo 6 da tabela 2). Apesar da forte associação, contudo, a inclusão da variável renda resultou em uma diminuição pequena da diferença entre os sexos nos modelos testados, passando as mulheres a apresentar uma chance $16 \%$ maior em relação aos homens de perceberem a sua saúde como ruim. A introdução da renda ocasionou também uma re- dução do efeito da variável idade do idoso.

No modelo 7 da tabela 2, foi introduzido o controle pelas doenças crônicas. As doenças crônicas foram fortemente relacionadas com a autopercepção de saúde dos idosos. Os idosos nas categorias "uma doença crônica" e "quatro ou mais doenças crônicas" tiveram um risco 1,86 e 11,98 vezes maior, respectivamente, de autoperceber a sua saúde como ruim em relação àqueles que não apresentavam doenças (categoria de referência). A entrada das doenças crônicas no modelo acarretou uma inversão nos diferenciais de sexo na autopercepção de saúde. As mulheres idosas passaram a ter uma chance aproximadamente $1 \%$ menor de relatar uma saúde ruim, mas esse resultado não foi estatisticamente significativo. Essa inversão poderia estar indicando uma associação entre as duas variáveis (sexo e doenças crônicas). Para testar essa hipótese, foi desenvolvido um modelo que incorpora a interação entre o sexo e as doenças crônicas, que será apresentado e discu- 
TABELA 3. Parâmetros e razão de chances (OR) para autopercepção ruim de saúde em idosos do Município de São Paulo (SP), Brasil, 2000 e 2001

\begin{tabular}{|c|c|c|}
\hline Variável & Parâmetros & OR \\
\hline Constante & $-0,317^{a}$ & \\
\hline \multicolumn{3}{|l|}{ Informante } \\
\hline Outro informante & 0,000 & 1,00 \\
\hline Entrevistado & $-0,203^{a}$ & 0,82 \\
\hline \multicolumn{3}{|l|}{ Sexo } \\
\hline Homens & 0,000 & 1,00 \\
\hline Mulheres & $-0,171^{a}$ & 0,84 \\
\hline \multicolumn{3}{|l|}{ Idade (anos) } \\
\hline 60 a 64 & 0,000 & 1,00 \\
\hline 65 a 69 & $0,229^{a}$ & 1,26 \\
\hline 70 a 74 & $-0,088^{a}$ & 0,92 \\
\hline 75 a 79 & $0,047^{a}$ & 1,05 \\
\hline 80 ou mais & $-0,394^{\mathrm{a}}$ & 0,67 \\
\hline \multicolumn{3}{|l|}{ Arranjo familiar } \\
\hline Mora acompanhado & 0,000 & 1,00 \\
\hline Mora sozinho & $-0,056^{a}$ & 0,95 \\
\hline \multicolumn{3}{|l|}{ Estado conjugal } \\
\hline Solteiro & 0,000 & 1,00 \\
\hline Casado/união consensual & $0,074^{a}$ & 1,08 \\
\hline Separado/divorciado & $0,081^{a}$ & 1,08 \\
\hline Viúvo & $-0,129^{a}$ & 0,88 \\
\hline \multicolumn{3}{|l|}{ Escolaridade } \\
\hline Sem escolaridade & 0,000 & 1,00 \\
\hline 1 a $4^{a}$ série (ensino fundamental) & $-0,327^{a}$ & 0,72 \\
\hline $5^{\mathrm{a}}$ série ou mais (ensino fundamental) & $-0,963^{a}$ & 0,38 \\
\hline \multicolumn{3}{|l|}{ Renda (salários mínimos) ${ }^{\mathrm{b}}$} \\
\hline$<1$ & 0,000 & 1,00 \\
\hline 1 a 3 & $0,298^{\mathrm{a}}$ & 1,35 \\
\hline 3 a 5 & $-0,023^{c}$ & 0,98 \\
\hline 5 ou mais & $-0,389^{a}$ & 0,68 \\
\hline \multicolumn{3}{|l|}{ Número de doenças crônicas ${ }^{d}$} \\
\hline Nenhuma & 0,000 & 1,00 \\
\hline 1 & $0,398^{a}$ & 1,49 \\
\hline 2 a 3 & $-0,819^{a}$ & 0,44 \\
\hline 4 ou mais & $2,354^{a}$ & 10,52 \\
\hline \multicolumn{3}{|l|}{ Capacidade funcionale } \\
\hline Independente & 0,000 & 1,00 \\
\hline Dependente nas AIVD & $0,915^{a}$ & 2,50 \\
\hline Dependente nas AVD/AIVD & $0,980^{\mathrm{a}}$ & 2,66 \\
\hline \multicolumn{3}{|l|}{ Interação (sexo $\times$ doenças crônicas) } \\
\hline Sexo masculino $\times$ sem doença crônica & 0,000 & 1,00 \\
\hline Sexo feminino $\times 1$ doença crônica & $0,375^{a}$ & 1,45 \\
\hline Sexo feminino $\times 2$ a 3 doenças crônicas & $-0,067^{c}$ & 0,94 \\
\hline Sexo feminino $\times 4$ doenças crônicas ou mais & $-0,065^{f}$ & 0,94 \\
\hline
\end{tabular}

Fonte dos dados básicos: Projeto Saúde, Bem-Estar e Envelhecimento na América Latina e Caribe (SABE) (15).

a $P<0,01$.

b Salário mínimo em abril de 2000: R\$151 (US\$ 89,93).

${ }^{c} P<0,05$.

${ }^{d}$ Hipertensão, artrite ou reumatismo, doença cardiovascular, diabetes, asma, bronquite ou enfisema, embolia ou acidente vascular cerebral e câncer.

e AIVD = atividades instrumentais de vida diária (preparar uma refeição quente e realizar tarefas domésticas leves e pesadas); $\mathrm{AVD}=$ atividades de vida diária (atravessar um cômodo da casa, comer, deitar-se e levantar da cama, usar o vaso sanitário, vestir-se e despir-se e tomar banho).

f $P<0,10$.

tido mais adiante. Embora o modelo em si não seja estatisticamente significativo, julgou-se importante ressaltar alguns dos seus impactos, que permanecem no modelo posterior, especificamente com relação à possibilidade de interação entre os efeitos das doenças crônicas e do sexo. Quando a variável capacidade funcional foi incluída (modelo 8 da tabela 2), as diferenças entre os sexos quanto à autopercepção de saúde se modificaram consideravelmente. As mulheres idosas passaram a ter uma chance $9 \%$ menor de se auto- avaliar como tendo uma saúde ruim em relação aos homens. Depois do controle por informante, sexo, idade, estado conjugal, arranjo familiar, escolaridade, renda e doenças crônicas, as mulheres idosas de ambos os níveis de capacidade funcional tiveram maior probabilidade de relatar uma boa saúde do que os homens.

A tabela 3 apresenta os resultados do modelo final, que incorpora uma variável resultante da interação entre o sexo e as doenças crônicas. Como a interação é estatisticamente significativa, os parâmetros e os valores de OR referentes a essas duas variáveis não podem mais ser interpretados separadamente. Os resultados dos parâmetros e das respectivas OR para a interação entre as variáveis são apresentados na tabela 4 .

Em relação aos homens idosos sem doenças crônicas, a chance de uma autopercepção ruim de saúde foi menor entre os idosos com duas a três doenças crônicas, tanto para as mulheres (65\%) quanto para os homens (56\%). Ainda tomando como referência os homens idosos sem doenças crônicas, verifica-se que a autopercepção ruim de saúde foi maior entre aqueles com uma doença crônica ( $49 \%$ no caso dos homens e $82 \%$ no caso das mulheres). A autopercepção ruim de saúde foi muitas vezes maior entre os idosos com quatro ou mais doenças crônicas, tanto do sexo masculino (9,5 vezes) quanto do sexo feminino ( 7,3 vezes). Tais resultados sugerem que, na ausência de doenças crônicas, ou na presença de duas ou mais doenças crônicas, as mulheres idosas auto-avaliam a sua própria saúde relativamente melhor do que os homens idosos.

\section{DISCUSSÃO}

Os resultados deste estudo revelaram que os idosos entrevistados avaliaram mais positivamente a própria saúde do que os idosos cuja avaliação foi realizada por outro informante. Essa diferença pode estar relacionada, por exemplo, com alterações de memória, que poderiam influenciar a capacidade do idoso de recordar e informar 
TABELA 4. Parâmetros e razão de chances (OR) para as interações entre o sexo e as doenças crônicas na autopercepção ruim de saúde em idosos do Município de São Paulo (SP), Brasil, 2000 e 2001

\begin{tabular}{lrrrrr}
\hline & \multicolumn{2}{c}{ Parâmetros } & & \multicolumn{2}{c}{ OR } \\
\cline { 2 - 3 } \cline { 5 - 6 } Número de doenças crônicas & Homens & Mulheres & & Homens & Mulheres \\
\hline Nenhuma & 0,000 & $-0,171$ & & 1,000 & 0,843 \\
1 & 0,398 & 0,602 & & 1,489 & 1,825 \\
2 a 3 & $-0,819$ & $-1,057$ & & 0,441 & 0,347 \\
4 ou mais & 2,354 & 2,118 & & 10,528 & 8,314 \\
\hline
\end{tabular}

${ }^{a}$ Hipertensão, artrite ou reumatismo, doença cardiovascular, diabetes, asma, bronquite ou enfisema, embolia ou acidente vascular cerebral e câncer.

adequadamente. Ainda, os idosos podem superestimar a sua condição de saúde para mostrar auto-suficiência, por medo de institucionalização ou de precisar de cuidados. Ademais, as diferenças culturais associadas com menores níveis de educação e renda e as expectativas mais baixas em relação ao estado de saúde do idoso podem interferir na avaliação de saúde feita por outro informante. De qualquer forma, trata-se de um resultado coerente, uma vez que a indicação de outro respondente para representar o entrevistado denota que o mesmo apresenta precariedade na sua condição de saúde.

Pode-se verificar que as mulheres idosas tiveram maior probabilidade de relatar uma boa autopercepção de saúde do que os homens. Contudo, esse efeito somente se sobressai após a inclusão da variável doenças crônicas no modelo e se acentua com a entrada da capacidade funcional. O presente estudo investigou os efeitos de interação entre as variáveis sexo e doenças crônicas e verificou a existência de uma interação significativa. Os resultados corroboram aqueles encontrados em outro estudo (20), que, contudo, não menciona um efeito de interação entre o sexo e as doenças crônicas. Essa interação pode ter três possíveis explicações. A primeira delas está associada aos novos papéis assumidos pelas mulheres na sociedade e na família, assim como às alterações em seu padrão de vida e escolaridade, que poderiam influenciar a forma como elas percebem a saúde. A segunda explicação refere-se à hipótese de que a contribuição, diferenciada por sexo, de diversas doenças crônicas e da capacidade funcional pode ser responsável pelas diferenças nos níveis de autopercepção da saúde. E, finalmente, a terceira explicação sugere que a mulher idosa que apresenta doenças crônicas e dependência funcional poderia estarse considerando saudável quando as doenças estão sob controle. Por outro lado, este estudo contraria outras investigações que mostraram uma melhor percepção de saúde por parte dos homens idosos em relação às mulheres $(21,22)$.

Com relação à variável renda, este estudo confirma os resultados encontrados em outras pesquisas $(23,24)$. Verifica-se uma importante relação da renda com a autopercepção de saúde. Os idosos com renda mais baixa apresentaram uma percepção ruim de saúde. Por outro lado, uma renda elevada foi um indicador robusto de uma boa autopercepção de saúde. A maior probabilidade de relatar saúde ruim entre os idosos com renda de 1 a 5 salários mínimos, em comparação com aqueles cujo rendimento era inferior a 1 salário mínimo, parece contraditória, mas remete ao debate acerca dos diferenciais de percepção segundo nível socioeconômico. Alguns autores (25, 26) já mostraram que a percepção de morbidade pode ser maior entre os estratos socioeconômicos menos favorecidos, o que pode estar relacionado, dentre outros aspectos, ao valor atribuído ao corpo, especialmente tendo em vista a necessidade de trabalhar, possivelmente mais premente entre os grupos de menor rendimento. Existem evidências também de que a baixa renda dos idosos atua negativamente no comportamento saudável, no ambiente domiciliar, no acesso aos serviços e aos cuidados de saúde, mesmo se esses são disponibilizados adequadamente, e, finalmente, nos recursos materiais. Há evidências de que os idosos mais pobres procuram menos os serviços de saúde, possuem baixa adesão aos tratamentos e têm pouco acesso aos medicamentos, o que reflete diretamente nas condições de saúde do indivíduo (27).

Os resultados do presente estudo indicam que a presença de doenças crônicas associada ao sexo é o mais forte determinante da autopercepção de saúde entre os idosos. A capacidade funcional demonstra também uma grande força explicativa. As associações encontradas foram coincidentes com as obtidas em estudos realizados em outros países (28-31). Conforme foi destacado na literatura, um maior número de doenças crônicas (quatro ou mais) implica o aumento da probabilidade de o idoso relatar uma percepção ruim de saúde. No entanto, observou-se neste estudo que os idosos pertencentes à categoria "duas a três doenças crônicas" tinham menos probabilidade de avaliar o seu estado de saúde como ruim. Tal resultado não era esperado, mas corrobora estudos anteriores (32) que encontraram uma boa autopercepção de saúde entre os indivíduos portadores de doenças crônicas. Uma possível explicação para esse efeito é que os idosos com duas ou três doenças crônicas podem se considerar saudáveis quando as doenças estão controladas.

Em relação à capacidade funcional, à medida que o grau de dependência aumenta, maior é a chance de o idoso autoperceber a sua saúde como ruim. Desse modo, a capacidade funcional passa a ser um dos principais determinantes da percepção de saúde do idoso e surge, portanto, como um novo paradigma de saúde, particularmente relevante para o idoso; num sentido amplo, seria o resultado do equilíbrio entre as várias dimensões da capacidade funcional do idoso (33).

Finalmente, os resultados do presente estudo indicam que, para se al- 
cançar um envelhecimento saudável, é preciso haver investimentos públicos efetivos no setor da saúde, no setor social e no setor econômico. As ações integradas que abordam, simultaneamente, os principais fatores determi- nantes da autopercepção de saúde podem contribuir significativamente para a promoção da saúde e do bemestar e, conseqüentemente, para a qualidade de vida dos idosos.

\section{REFERÊNCIAS}

1. Carvalho JAM, Garcia RA. O envelhecimento da população brasileira: um enfoque demográfico. Cad Saude Publica. 2003;19(3): 109-18.

2. Chaimowicz F. A saúde dos idosos brasileiros às vésperas do século XXI: problemas, projeções e alternativas. Rev Saude Publica. 1997; 31(2):184-200.

3. World Health Organization. Men, ageing and health-achieving health across the life span. Genebra: WHO, Noncommunicable Diseases Prevention and Health Promotion Department; 2001. (WHO/NMH/NPH/01.2).

4. Ramos LR. A explosão demográfica da terceira idade no Brasil: uma questão de saúde pública. Gerontologia. 1993;1(1):3-8.

5. Portrait F, Lindeboom M, Deeg D. Life expectancies in specific health states: results from a joint model of health status and mortality of older persons. Demography. 2001;38(4): 525-36.

6. Bailis DS, Segall A, Chipperfield JG. Two views of self-rated general health status. Soc Sci Med. 2003;56(2):203-17.

7. Guccione AA. Fisioterapia geriátrica. 2aㅡ ed. Rio de Janeiro: Guanabara Koogan; 2002.

8. Martikainen P, Aromaa A, Heliovaara M, Klaukka T, Knekt P, Maatela J, et al. Reliability of perceived health by sex and age. Soc Sci Med. 1999;48(8):1117-22.

9. Ofstedal MB, Zimmer Z, Cruz G, Chan A, Lin YH. Self-assessed health expectancy among older Asians: a comparison of Sullivan and multistate life table methods. Ann Arbor: University of Michigan, Population Studies Center; 2002. (Research Reports/Population Studies Center 03-60).

10. Kaplan GA, Camacho T. Perceived health and mortality: a nine-year follow-up of the human population laboratory cohort. Am J Epidemiol. 1983;117(3):292-304.

11. Idler EL, Benyamini Y. Self-rated health and mortality: a review of twenty-seven community studies. J Health Soc Behav. 1997;38(1): 21-37.

12. Marcellini F. Health perception of elderly people: the results of a longitudinal study. Arch Gerontol Geriatr Suppl. 2002;35(Suppl): 181-9.
13. Appels A, Bosma H, Grabauskas V, Gostautas A, Sturmans F. Self-rated health and mortality in a Lithuanian and a Dutch population. Soc Sci Med. 1996;42(5):681-9.

14. Alves LC. Determinantes da autopercepção de saúde dos idosos do município de São Paulo, 1999/2000 [dissertação de mestrado]. Belo Horizonte: Universidade Federal de Minas Gerais, Centro de Desenvolvimento e Planejamento Regional; 2004.

15. Peláez M, Palloni A, Albala C, Alfonso JC, Ham-Chande R, Hennis A, et al. Survey on aging, health and wellbeing, 2000. Washington, D.C.: Pan American Health Organization, World Health Organization; 2003.

16. Instituto Brasileiro de Geografia e Estatística. Pesquisa Nacional por Amostra de Domicílios (PNAD 98). Rio de Janeiro: IBGE; 1998.

17. Folstein MF, Folstein S, McHugh PR. "MiniMental State." A practical method for grading the cognitive state of patients for the clinician. J Psychiatr Res. 1975;12(3):189-98.

18. Icaza MG, Albala C. Mini-mental State Examination (MMSE): el estudio de demencias en Chile. Santiago: Organización Panamericana de la Salud; 1999. JM, Filos S. Measurement of functional activities in older adults in the community. J Gerontol. 1982;37(3):323-9.

20. Arber S, Cooper H. Gender differences in health in later life: the new paradox? Soc Sci Med. 1999;48(1):61-76.

21. Kubzansky LD, Berkman LF, Glass TA, Seeman TE. Is educational attainment associated with shared determinants of health in the elderly? Findings from the MacArthur studies of successful aging. Psychosom Med. 1998; 60(5):578-85.

22. McDonough $P$, Walters V. Gender and health: reassessing patterns and explanations. Soc Sci Med. 2001;52(4):547-59.

23. Arber S, Ginn J. Gender and inequalities in health in later life. Soc Sci Med. 1993;36(1): 33-46.

24. Zimmer Z, Amornsirisomboon P. Socioeconomic status and health among older adults in Thailand: an examination using multiple indicators. Soc Sci Med. 2001;52(8):1297-311.
19. Pfeffer RI, Kurosaki TT, Harrah CH Jr, Chance
Agradecimentos. Os autores agradecem o apoio do Conselho Nacional de Desenvolvimento Científico e Tecnológico (CNPq), Brasil, e a contribuição dos pareceristas anônimos à versão preliminar deste artigo.
25. Rodrigues RN. Determinantes sócio-econômicos de morbidade e mortalidade numa área de industrialização recente: o caso de Belo Horizonte [dissertação de mestrado]. Belo Horizonte: Universidade Federal de Minas Gerais, Centro de Desenvolvimento e Planejamento Regional; 1981.

26. Rodrigues RN. "Vida severina", healthy family?: morbidity and mortality in two metropolitan regions of Brazil [tese]. Canberra: Australian National University; 1989.

27. Lima-Costa MFF, Barreto SM, Giatti L, Uchoa E. Desigualdade social e saúde entre idosos brasileiros: um estudo baseado na Pesquisa Nacional por Amostra de Domicílios. Cad Saude Publica. 2003;19(3):745-57.

28. Ferraro KF, Farmer MM, Wybraniec JA. Health trajectories: long-term dynamics among black and white adults. J Health Soc Behav. 1997;38(1):38-54.

29. Hoeymans N, Feskens EJM, Kromhout D, Van Den Bos GAM. Ageing and the relationship between functional status and self-rated health in elderly men. Soc Sci Med. 1997; 45(10):1527-36.

30. Damián J, Ruigómez A, Pastor V, MartínMoreno JM. Determinants of self assessed health among Spanish older people living at home. J Epidemiol Community Health. 1999; 53(7):412-6.

31. Gama EV, Damián J, Molino JP, López MR, Pérez ML, Iglesias FJG. Association of individual activities of daily living with self-rated health in older people. Age Ageing. 2000; 29(3):267-70.

32. Cott CA, Gignac MAM, Badley EM. Determinants of self rated health for Canadians with chronic disease and disability. J Epidemiol Community Health. 1999;53(11):731-6.

33. Ramos LR. Fatores determinantes do envelhecimento saudável em idosos residentes em centro urbano: Projeto Epidoso, São Paulo. Cad Saude Publica. 2003;19(3):793-8.

Manuscrito recebido em 29 de junho de 2004. Aceito em versão revisada em 28 de setembro de 2004. 
ABSTRACT Objectives. To investigate the influence that demographic determinants, socioeconomic determinants, chronic diseases, and functional capacity have on self-rated health among elderly persons (60 years and older) living in the city of São Paulo, São Paulo, Brazil, and to investigate the existence of differences between men and women in terms of their self-rated health.

Determinants of self-rated health among elderly persons in São Paulo, Brazil

Methods. The study was carried out using data collected in the city of São Paulo as part of a project called Health, Well-being, and Aging in Latin America and the Caribbean (the "SABE project"). We analyzed data on 2135 elderly individuals $(58.6 \%$ women; mean age, 69.4 years; median age, 68.0 years). The dependent variable was self-rated health (good or poor). The following independent variables were considered: (1) demographic ones (age, sex, marital status, and living arrangements (whether the elderly person lived alone or with others)), (2) socioeconomic ones (schooling and income), (3) the number of chronic diseases (hypertension, arthritis or rheumatism, cardiovascular disease, diabetes, asthma, bronchitis or emphysema, embolism or stroke, and cancer), and (4) functional capacity. To estimate the association between self-rated health and the independent variables and to study gender differences, a multiple binary logistic regression analysis was performed.

Results. The presence of chronic diseases in association with gender was the strongest determinant of self-rated health among the elderly in São Paulo. Among men with four or more chronic diseases, they were 10.53 times as likely to characterize their health as poor; among women with four or more chronic diseases, the ratio was 8.31. Functional capacity, schooling, and income were also strongly associated with self-rated health, and the influence of age was significant. The elderly women were more likely to report good self-rated health than were men when the women or men either had no chronic diseases or had two or more.

Conclusions. Our results indicate the need for simultaneous, comprehensive actions in the health sector, social services, and the economic sector to address the main determinants of self-rated health in order to promote well-being and quality of life among the elderly.

Keywords Chronic disease, sex factors, health services for the aged. 